\title{
Fiber laser sensor system based on a random mirror and a compound ring resonator for displacement measurements
}

\author{
S. Rota-Rodrigo*1 ${ }^{1}$, M. González-Herráez ${ }^{2}$, M. Lopez-Amo ${ }^{1}$ \\ ${ }^{1}$ Departamento de Ingeniería Eléctrica y Electrónica, Universidad Pública de Navarra, Campus de Arrosadía, \\ 31006 Pamplona - Navarra, Spain. \\ ${ }^{2}$ Departamento de Electrónica, Universidad de Alcalá, Edificio Politécnico, Madrid 28871, Spain.
}

\begin{abstract}
In this work, we present a random laser sensor system for increasing simultaneously the sensitivity and interrogation stability of a fiber-optic displacement sensor. The system is based on a random distributed fiber laser modulated by a double-coupler ring resonator within which is placed the sensor. This allows to increase the sensitivity of the sensor and also its interrogation stability. The experimental results show a dynamic range increment (and consequently its sensitivity) of $10 \mathrm{~dB}$. Moreover, by using this laser-based interrogation system, the instability is reduced to $0.04 \mathrm{~dB}$. In addition, wavelength filtering elements are not required, simplifying the system.
\end{abstract}

Keywords: Optical fiber sensor, fiber laser sensor, laser ring resonator, random laser.

\section{INTRODUCTION}

Random distributed feedback (RDFB) fiber lasers have received increased attention in recent years, due to their advantages such as stability and wide band operation. RDFB lasers are based on the combination of the Rayleigh scattering feedback together with amplification, generally through the Raman effect [1]. A RDFB laser is formed at least by one random mirror that can be achieved by a long distance of SMF like in [2], or by using a fiber that increases the scattering and the nonlinearities like a dispersion-compensating fiber (DCF). This allows reducing significantly the required length of fiber up to a few $\mathrm{km}$ [3]. The second mirror can be used to modify the RDFB spectrum in order to achieve a multi-wavelength laser, as in [4] where a photonic crystal fiber loop mirror is used for this purpose, or in [5] where a mirror structure including a modulator was used for the internal modulation of the RDFB laser.

In this paper, we use a fiber optical ring resonator (FORR) to achieve a modulation of the spectral response of the RDFB. In terms of sensing, FORRs provide strong attenuation sensitivity at the expense of a periodical response in frequency. The period of the FORR frequency response is inversely proportional to its length. FORR is an interesting structure in combination with intensiometric sensors because it allows a substantial increase in the sensitivity of the inserted sensors into the ring, due to the multiple recirculations of the light through the sensors. It is important to note that this kind of sensors can be used for measuring physical [6] as well as chemical [7] parameters.

There are different previous works based on FORR that use diverse techniques of interrogation as for example a sweep interrogation with a tunable laser or using a vector analyzer [8], or the cavity ring-down (CRD) technique [9] that analyzes the light intensity decaying of an input pulse inside the cavity. Both options require expensive equipment, in the optical domain for the first case, and in the electrical domain in the CRD case. Turning the resonator into a laser cavity is a way to simplify the interrogation method and increase the stability of the measurement, as in our previous work [8], where we demonstrated that using an erbium doped fiber ring laser generated by using the FORR sensor as seed, the instability of the system could be improved 100 times in comparison with an external interrogation [10]. 
In this paper we present a RDFB laser sensor systems based on a compound FORR for increasing simultaneously the sensitivity and stability of an intensiometric displacement fiber sensor. The FORR is formed by a double coupler configuration, whose transmitted and reflected signals show complementary responses. The self-interrogator random fiber laser was obtained by using the FORR reflected signal as filter mirror, a $2.5 \mathrm{Km}$ DCF as distributed mirror and using the Raman effect within the DCF as amplification method.

\section{EXPERIMENTAL SET UP AND RESULTS}

Figure 1 depicts the schematic setup of the random fiber laser sensor system. The gain is based on the Raman effect, and was achieved by forward pumping a $2.4 \mathrm{Km}$ dispersion-compensating fiber with a laser at $1445 \mathrm{~nm}$ (RLD-3-1445) and a pump power of $1.2 \mathrm{~W}$. The DCF also acts as a distributed mirror for generating the random laser. The second mirror was formed by the combination of a circulator and the compound FORR. The resonator consists of a double coupler configuration within which the displacement sensor (Fig. 3a) and a polarization control (PC) are placed. This double coupler configuration allows the extraction of the FORR complementary response: while the transmission displays periodic notches in the spectrum (with a periodicity given by $c / n L$ ), the reflection shows the complementary periodic resonances with equal power sensitivity and opposite sign [10]. The reflection response is thus used, to achieve the optimum overall power sensitivity in the output port. The total length of the FORR was about 2 m consequently, a resonance peaks separation of about $3 \mathrm{pm}$ might be expected.

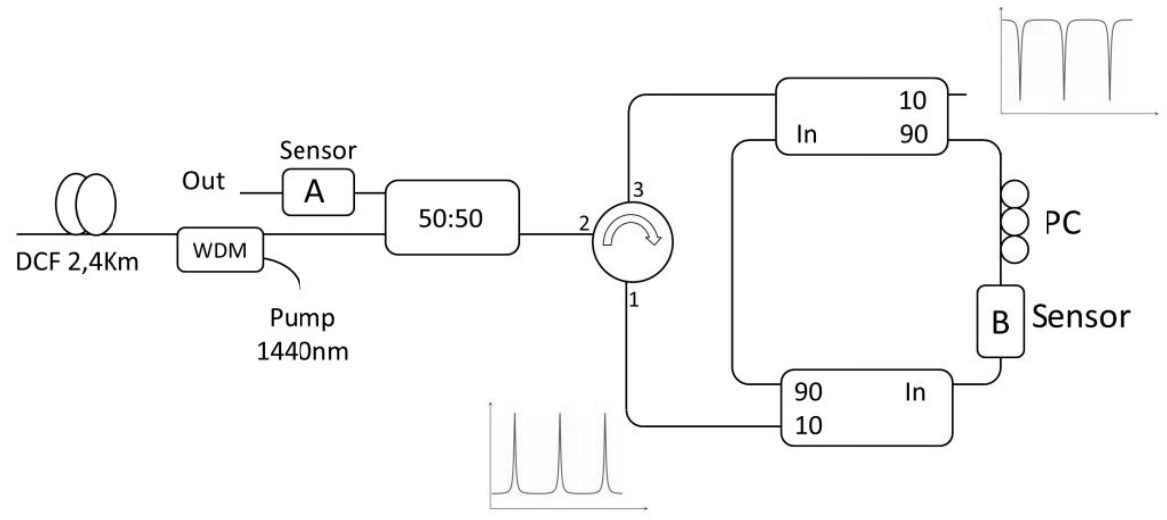

Figure 1 - Experimental setup of the micro-bending random fiber laser sensor system.

Figure 2 shows the optical spectrum of the random laser sensor system output measured with a high resolution (0.08pm) optical spectrum analyzer (BOSA OPT100). As it can be seen, the response of the laser is modulated by the periodical response of the FORR, with a peaks separation of $0.3 \mathrm{pm}$, in good agreement with the theoretical value for a $2 \mathrm{~m}$ ring resonator. To achieve a cost-effective interrogation of the sensor, an optical power meter (Ando Aq2140) was used in the output port, this way the total output power was integrated avoiding the instabilities of each resonance.

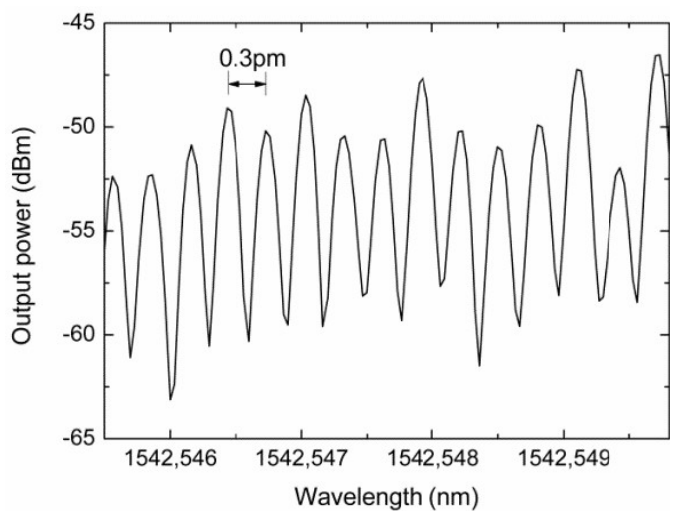

Figure 2 - Optical spectrum of the random fiber laser sensor system measured with a BOSA. 
The displacement sensor system characterization was conducted by using a precision mechanic attenuator based on stress-inducing plates (Fig. 3a), similar to the one used in [11]. In order to determine the improvement achieved by using the compound FORR, the sensor was placed alternatively in positions outside the ring (A) and inside it (B) (Fig. 1). The measurements were carried out in the range from 0 to $1000 \mu \mathrm{m}$ with a step of $4.27 \mu \mathrm{m}$. Before each measurement, the polarization was optimized using the PC. Figure $3 \mathrm{~b}$ shows that, when the sensor is located inside the ring (Position B), a dynamic range increment of $10 \mathrm{~dB}$ at $400 \mu \mathrm{m}$ was achieved. This configuration allows to measure up to $780 \mu \mathrm{m}$ of displacement.
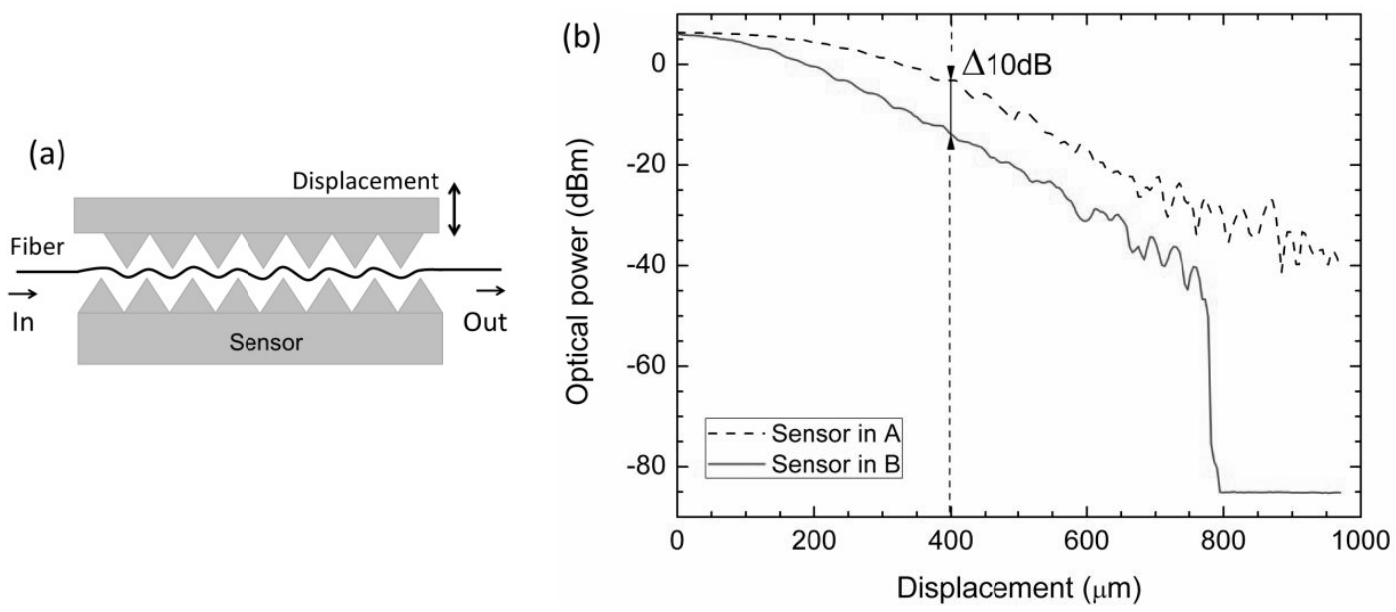

Figure 3 - (a) Schematic of the displacement sensor based on bending. (b) Output power response in function of the sensor displacement in positions A (dotted) and B (continuous).

An instability measure was also carried out at a displacement of $400 \mu \mathrm{m}$. The measurement was performed along 30 minutes, with samples each 10 seconds. Figure 4 shows the power fluctuations, showing a maximum instability of 0.04 $\mathrm{dB}$, which is considerable better than the $0.21 \mathrm{~dB}, 0.34 \mathrm{~dB}$ and $1.48 \mathrm{~dB}$ that were previously achieved by using an ORR in combination with different EDFRLs in [10].

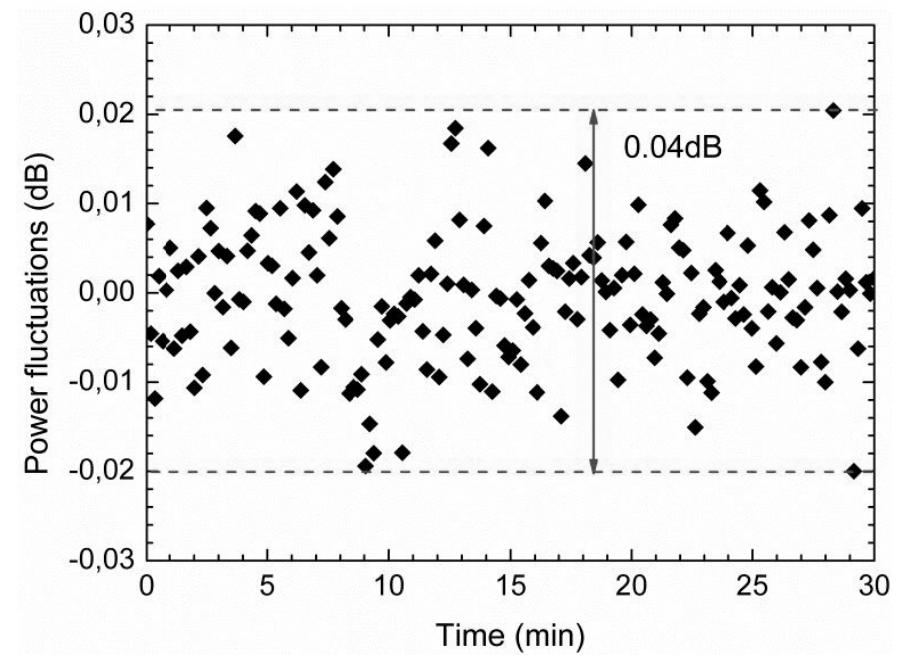

Figure 4 - Output power fluctuations of the system for a displacement of $400 \mu \mathrm{m}$.

\section{CONCLUSIONS}

In conclusion, in this paper a new sensor system for enhancing a fiber optic intensity sensor is presented. The system consists of a random distributed feedback laser generated by using a forward pumped DCF that also acts as random mirror. The second mirror is formed by a double-coupler ring resonator, within which is allocated the intensity sensor. 
The main advantages of this method are the ability to increase the dynamic range of the intensity sensor up to $10 \mathrm{~dB}$ and also, because the system works as a self-interrogator of the sensor, it improves the instability up to $0.04 \mathrm{~dB}$. The system has been experimentally demonstrated for a displacement sensor, but the proposed interrogation system is suited for any kind of transmissive intensity sensors.

\section{ACKNOWLEDGMENTS}

The authors would like to acknowledge the financial support from the Spanish Ministerio de Educación y Ciencia through projects TEC2013-47264-C2-2-R, TEC2012-37958-C02-01 and Innocampus; from the European FEDER funding, ECOAL-MGT SUDOE project and from the European Research Council through Starting Grant U-FINE (Grant No. 307441).

\section{REFERENCES}

[1] Ania-Castañon, J. D., Ellingham, T. J., Ibbotson, R., Chen, X., Zhang, L. and Turitsyn, S. K. "Random distributed feedback fibre laser," Phys. Rev. Lett. 96, 023902 (2006).

[2] Zhu, Y.Y., Zhang, W.L. and Jiang, Y. "Tunable multi-wavelength fiber laser based on random Rayleigh backscattering," IEEE Phot. Technol. Lett., 25(16), (2013).

[3] Fernandez-Vallejo, M., Rota-Rodrigo, S. and Lopez-Amo, M. "Comparative study of ring and random cavities for fiber lasers," App. Opt. 53(16), (2014).

[4] Pinto, A. M. R., Frazão, O., Santos, J. L. and Lopez-Amo, M. "Multiwavelength raman fiber laser using Hi-Bi photonic crystal fiber loop mirrors combined with random cavities," J. of Lightwave Technol. 29(10), (2011).

[5] Bravo, M., Fernandez-Vallejo, M. and M. Lopez-Amo, "Internal modulation of a random fiber laser," Opt. Lett. ,38(9), (2013).

[6] Zhang, L., Lu, P., Chen, L., Huang, C., Liu, D. and Jiang, S. "Optical fiber strain sensor using fiber resonator based on frequency comb Vernier spectroscopy," Opt. Lett. 37, 2622-2624, (2012).

[7] Guo, Y. and Fan, X. “Optofluidics in Bio-Chemical Analysis,” Proc. SPIE, 8212, 82120F, (2012).

[8] Bravo, M., Angulo-Vinuesa, X., Martin-Lopez, S., Lopez-Amo, M. and Gonzalez-Herraez, M. "Slow-light and enhanced sensitivity in a displacement sensor using a lossy fiber-based ring resonator," J. Lightwave. Technol. 31, 3752-3757, (2013).

[9] Wang, C. "Fiber Loop Ringdown- a Time-Domain Sensing Thecnique for Multi-Function Fiber Otpic Sensor Platforms: Current Status and Design Perspectives," Sensors 9, 7595-7621, (2009)

[10] Rota-Rodrigo, S., González-Herráez, M. and López-Amo, M. " Compound lasing fiber optic ring resonators for sensign," Proc. SPIE 9157, paper 9157D7, (2014).

[11] Bravo, M., Pinto, A. M. R., Lopez-Amo, M., Kobelke, J. and Shuster, K. "High precision micro-displacement fiber sensor through a suspended-core Sagnac interferometer," Opt. Lett. 37, (2012). 